\title{
Theoretical Analysis of Two Collinear Cracks in an Orthotropic Solid under Linear Thermal Flux and Linear Mechanical Load
}

\author{
Bing Wu $\mathbb{D}^{1},{ }^{1}$ Bao-Yin Zhu, ${ }^{2}$ Hao-Meng Song, ${ }^{1}$ and Qiong-Ao Huang $\mathbb{D}^{3}$ \\ ${ }^{1}$ Technology Innovation Center for Testing and Evaluation in Civil Engineering of Hebei Province, Hebei University, Baoding, \\ Hebei 071002, China \\ ${ }^{2}$ Suzhou Nuclear Power Research Institute, Xihua Road 1688, Suzhou 215004, China \\ ${ }^{3}$ South China Research Center for Applied Mathematics and Interdisciplinary Studies, South China Normal University, \\ Guangzhou 510631, China
}

Correspondence should be addressed to Qiong-Ao Huang; huangqiongao@m.scnu.edu.cn

Received 27 August 2021; Revised 7 November 2021; Accepted 2 December 2021; Published 9 February 2022

Academic Editor: David Carf

Copyright (c) 2022 Bing Wu et al. This is an open access article distributed under the Creative Commons Attribution License, which permits unrestricted use, distribution, and reproduction in any medium, provided the original work is properly cited.

\begin{abstract}
This paper studies the problem of a cracked orthotropic solid subject to linear thermal flux and linear mechanical load. The proposed extended partially insulated crack model is employed to simulate two collinear cracks. Taking advantage of Fourier transform technique and superposition theory, the closed form of some physical quantities and fracture parameters is obtained. Some simple examples are employed to demonstrate dimensionless thermal conductivity $\left(R_{c}\right)$ between the upper and below crack regions, and the proposed coefficient $(\varepsilon)$ has great effects on some physical quantities and fracture parameters.
\end{abstract}

\section{Introduction}

Multicomponent composite materials are widely used in the material industry. However, considering the complex factors involving working environment, internal and external loads, and production process, it is inevitable to contain a series of various cracks in these solids. The appearances of different kinds of cracks will reduce the capacity of cracked structures and even bring about severe accidents. Therefore, it is vital to do some research on fracture analysis of a cracked solid by utilizing the theory of thermal elasticity for the purpose of safety [1-3]. With the rapid growth of thermoelasticity theory, a great deal of treatises and papers was published to investigate fracture characters of cracked solids [4-6]. The fracture parameters of an orthotropic material containing a central crack under heat flow were obtained by Tsai [7]. The closed form of fracture parameters of cracked orthotropic solids was calculated by Ju and Rowlands [8]. The closed form of some physical quantities of two collinear cracks was studied by Chen and Zhang [9]. The transient thermal problem of a cracked orthotropic plate was taken into account by Noda [10]. Some physical quantities of a cracked orthotropic semi-infinite medium were given by Rizk [11]. On the other hand, the thermoelastic problems of orthotropic functionally graded solids brought about the widespread attention. For example, the fracture parameters of orthotropic functionally graded solids under mechanical load were given explicitly by Kim and Paulino [12]. The problem of a cracked solid subject to plane temperaturestep waves was investigated by Brock [13]. The equivalent domain integral was formulated to study the fracture problems subject to thermal stresses by Dag [14]. The problems of cracked orthotropic solids subject to symmetrical thermomechanical loads with application of Fourier transform technique (FTT) and superposition principle were studied by $\mathrm{Wu}$ et al. [15].

Subject to thermal load, the analysis of fracture behavior for cracked solids which were often regarded as orthotropic or isotropic has generated enormous publicity [16-20]. To 
simulate two collinear cracks, a partially insulated crack model prevailed [21-23].

$$
Q_{1 c}=-h_{c} \Delta T
$$

where the definitions of $Q_{1 c}, h_{c}$, and $\Delta T$ have been given in detail [3]. The case of $h_{c} \longrightarrow 0$ or $h_{c} \longrightarrow \infty$ denotes a fully thermally impermeable or permeable state.

The following extended partially insulated crack model is also put forward by virtue of mathematical intuition.

$$
Q_{1 c}=-h_{c} \Delta T+\varepsilon Q_{1},
$$

where $Q_{1}$ presents initial heat flux. The coefficient $\varepsilon$ is considered a constant. Whether it is negative or positive is mainly relies on the portion of thermal flux and mechanical load. Clearly, the crack model proposed in (2) returns to (1) when $\varepsilon=0$.

The reasons of introducing constant $\varepsilon Q_{1}$ in (2) are as follows. First, the value of $h_{c}$ does not precisely address the cracks with thermal resistance. Second, the constant $\varepsilon Q_{1}$, which is introduced as an adjustment factor, conforms to the complex situation and meets the abnormal state of crack surface.

This paper employs an extended partially insulated crack model to discuss two collinear cracks under linear thermal flux and linear mechanical load. The thermoelastic field is given in explicit form based on the proposed extended partially insulated crack model, Fourier transform, and superposition theory. The results show the effects of dimensionless thermal conductivity $\left(R_{c}\right)$ between the upper and below crack regions and the proposed coefficient $(\varepsilon)$ on $Q_{1 c}$ and $K_{I I}$ and $S$. It is revealed the boundary conditions of crack surface, thermal properties of crack, and the raised coefficient should be paid attention to the analysis of crack growth under thermal load in numerical results.

\section{Problem Statement}

Two collinear cracks in an orthotropic solid are taken into account as shown in Figure 1. They are located at $a<|x|<b$.

Making use of the state of plane stress [3], we obtain

$$
\begin{gathered}
\sigma_{x}=c_{11} \frac{\partial u}{\partial x}+c_{12} \frac{\partial v}{\partial y}-\beta_{1} T, \\
\sigma_{y}=c_{12} \frac{\partial u}{\partial x}+c_{22} \frac{\partial v}{\partial y}-\beta_{2} T, \\
\tau_{x y}=c_{66}\left[\frac{\partial u}{\partial y}+\frac{\partial v}{\partial x}\right],
\end{gathered}
$$

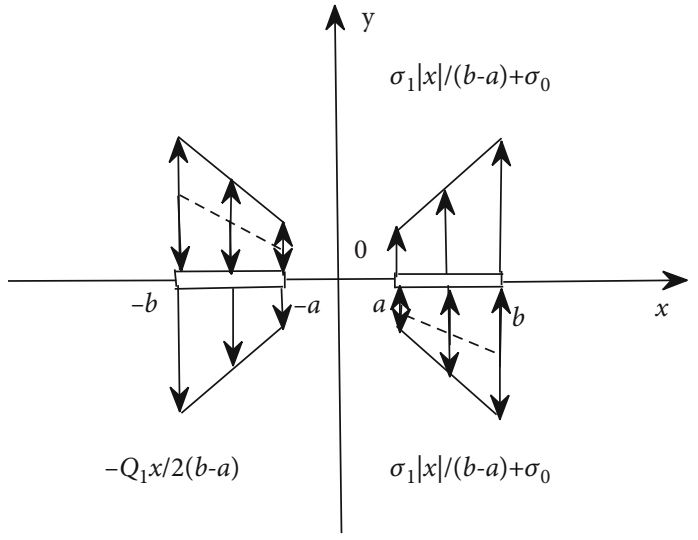

FIgURe 1: Two collinear cracks subject to linear thermal flux and linear mechanical load.

where

$$
\begin{gathered}
c_{11}=\frac{E_{x x}}{1-v_{x y} v_{y x}}, \\
c_{22}=\frac{E_{y y}}{1-v_{x y} v_{y x}}, \\
c_{12}=\frac{E_{x x} v_{y x}}{1-v_{x y} v_{y x}}=\frac{E_{y y} v_{x y}}{1-v_{x y} v_{y x}}, \\
{\left[\begin{array}{c}
\beta_{1} \\
\beta_{2}
\end{array}\right]=\left[\begin{array}{ll}
c_{11} & c_{12} \\
c_{12} & c_{22}
\end{array}\right]\left[\begin{array}{l}
\alpha_{x x} \\
\alpha_{y y}
\end{array}\right],}
\end{gathered}
$$

where the definitions of $u, v, \sigma_{x}, \sigma_{y}, \tau_{x y} T, v_{x x}, v_{y y} E_{x x}, E_{y y}$, $c_{66}=G_{x y}, \alpha_{x x}$, and $\alpha_{y y}$ have been given in [3].

$$
\begin{aligned}
& \frac{\partial \sigma_{x}}{\partial x}+\frac{\partial \tau_{x y}}{\partial y}=0 \\
& \frac{\partial \tau_{x y}}{\partial x}+\frac{\partial \sigma_{y}}{\partial y}=0 .
\end{aligned}
$$

One obtains

$$
\begin{gathered}
c_{11} \frac{\partial^{2} u}{\partial x^{2}}+c_{66} \frac{\partial^{2} u}{\partial y^{2}}+\left(c_{12}+c_{66}\right) \frac{\partial^{2} v}{\partial x \partial y}=\beta_{1} \frac{\partial T}{\partial x}, \\
c_{66} \frac{\partial^{2} v}{\partial x^{2}}+c_{22} \frac{\partial^{2} v}{\partial y^{2}}+\left(c_{12}+c_{66}\right) \frac{\partial^{2} u}{\partial x \partial y}=\beta_{2} \frac{\partial T}{\partial y} .
\end{gathered}
$$

Making use of the Fourier heat conduction leads to

$$
\begin{aligned}
& Q_{x}=-\lambda_{x} \frac{\partial T}{\partial x} \\
& Q_{y}=-\lambda_{y} \frac{\partial T}{\partial y}
\end{aligned}
$$

where the definitions of $Q_{x}, Q_{y}, \lambda_{x}$, and $\lambda_{y}$ have been given in [3]. Furthermore, based on the equilibrium equation, 
one has

$$
\frac{\partial Q_{x}}{\partial x}+\frac{\partial Q_{y}}{\partial y}=0
$$

Taking advantage of the thermal equilibrium equations brings out

$$
\lambda^{2} \frac{\partial^{2} T}{\partial x^{2}}+\frac{\partial^{2} T}{\partial y^{2}}=0
$$

where

$$
\lambda=\sqrt{\frac{\lambda_{x}}{\lambda_{y}}}
$$

The crack face boundary conditions are depicted as

$$
\begin{gathered}
Q_{y}^{I I}(x, 0)-Q_{y}^{I}(x, 0)=-\frac{Q_{1} x}{2(b-a)^{2}}, \quad|a|<x<|b|, \\
\sigma_{y}^{I I}(x, 0)=\sigma_{y}^{I}(x, 0)=\frac{\sigma_{1}|x|}{b-a}+\sigma_{0}, \quad|a|<x<|b|,
\end{gathered}
$$

Hereafter, the subscript ' $I$ ' or ' $I I$ ' denotes the physical quantity of the upper $(y>0)$ or below $(y<0)$ part. $Q_{0}, Q_{1}$, $\sigma_{0}$, and $\sigma_{1}$ stand for the prescribed constants. Based on (14) and (15), thermal flux is composed of only one part $\left(-Q_{1} x / 2(b-a)^{2}\right)$ and mechanical loading is divided into two parts $\left(\sigma_{1}|x| /(b-a)\right.$ and $\left.\sigma_{0}\right)$. As linear thermal flux and linear mechanical load are antisymmetrical and symmetrical, respectively, the thermoelastic field of the region $(x>0)$ is only dealt with. The crack-surface boundary conditions are expressed with the application of the improved partially insulated crack model.

$$
\begin{gathered}
\tau_{x y}^{I I}(x, 0)=\tau_{x y}^{I}(x, 0)=0, \quad a<x<b, \\
Q_{y}^{I I}(x, 0)-Q_{y}^{I}(x, 0)=-\frac{Q_{1}-Q_{1 c}}{2(b-a)^{2}} x \quad a<x<b, \\
\sigma_{y}^{I I}(x, 0)=\sigma_{y}^{I}(x, 0)=\frac{\sigma_{1} x}{b-a}+\sigma_{0}, \quad a<x<b .
\end{gathered}
$$

According to Equations (17) and (18), the solutions under thermal flux $\left(-Q_{1} x / 2(b-a)^{2}\right)$ and mechanical loading $\left(\sigma_{0}\right)$ have been given explicitly in $[24,25]$. Next, we depict the boundary conditions of crack-surface subject to linear mechanical load $\left(\sigma_{1} x /(b-a)\right)$.

$$
\begin{aligned}
& \tau_{x y}^{I}(x, 0)=\tau_{x y}^{I I}(x, 0)=0, \quad a<x<b, \\
& \sigma_{y}^{I I}(x, 0)=\sigma_{y}^{I}(x, 0)=\frac{\sigma_{1} x}{b-a}, \quad a<x<b,
\end{aligned}
$$

where

$$
Q_{1 c}=-h_{c}\left(T^{I}(x, 0)-T^{I I}(x, 0)\right)+\varepsilon Q_{1} .
$$

Besides, some physical quantities conform to the following conditions:

$$
\begin{array}{cc}
\tau_{x y}^{I}(x, 0)=\tau_{x y}^{I I}(x, 0), \sigma_{y}^{I}(x, 0)=\sigma_{y}^{I I}(x, 0), & x>b \text { or } 0<x<a, \\
u^{I}(x, 0)=-u^{I I}(x, 0), v^{I}(x, 0)=-v^{I I}(x, 0), & x>b \text { or } 0<x<a, \\
T^{I}(x, 0)=T^{I I}(x, 0), Q_{y}^{I}(x, 0)=Q_{y}^{I I}(x, 0), & x>b \text { or } 0<x<a .
\end{array}
$$

\section{Solution Procedure}

3.1. Temperature Field. According to [25], one obtains the explicit form of temperature difference on crack faces as

$T^{I}(x, 0)-T^{I I}(x, 0)=-\frac{Q_{1}-Q_{1 c}}{2(b-a)^{2} \lambda \lambda_{y}} \sqrt{\left(x^{2}-a^{2}\right)\left(b^{2}-x^{2}\right)}, \quad a<x<b$.

3.2. Elastic Field. To achieve the goal of explicit form in Equations (8) and (9), $u^{I, I I}(x, y)$ and $v^{I, I I}(x, y)$ are expressed according to [26].

$$
u^{I, I I}(x, y)=\sum_{i=1}^{2} u_{i}^{I, I I}(x, y), \quad v^{I, I I}(x, y)=\sum_{i=1}^{2} v_{i}^{I, I I}(x, y),
$$

where the definitions of $u_{j}^{I, I I}(x, y)$ and $v_{j}^{I, I I}(x, y)(j=1,2)$ have been given in [26].

$$
\begin{gathered}
u_{1}^{I, I I}(x, y)=\sum_{j=1}^{2} \int_{0}^{+\infty} g_{j}^{I, I I}(\xi) e^{-\xi \delta^{ \pm} \gamma_{j} y} \sin (\xi x) d \xi, \\
v_{1}^{I, I I}(x, y)=\sum_{j=1}^{2} \int_{0}^{+\infty} \eta_{j} \delta^{ \pm} g_{j}^{I, I I}(\xi) e^{-\xi \delta^{ \pm} \gamma_{j} y} \cos (\xi x) d \xi .
\end{gathered}
$$

Hereafter, $\delta^{+}=1$ or $\delta^{-}=-1$ denotes $y>0$ or $y<0 . g_{j}^{I, I I}$ ( $\xi$ ) need to solve. The definitions of $\gamma_{j}(j=1,2)$ have been given in [26].

$$
c_{22} c_{66} \gamma^{4}+\left(c_{12}^{2}+2 c_{12} c_{66}-c_{12} c_{22}\right) \gamma^{2}+c_{11} c_{66}=0,
$$

where

$$
\eta_{j}=\frac{c_{11}-c_{66} \gamma_{j}^{2}}{\left(c_{12}+c_{66}\right) \gamma_{j}}
$$

Furthermore, $u_{2}^{I, I I}(x, y)$ and $v_{2}^{I, I I}(x, y)$ are chosen as

$$
\begin{gathered}
u_{2}^{I, I I}(x, y)=\sum_{j=1}^{2} \int_{0}^{+\infty} g^{* I, I I}(\xi) e^{-\delta \pm \xi \lambda y} \sin (\xi x) d \xi, \\
v_{2}^{I, I I}(x, y)=\sum_{j=1}^{2} \int_{0}^{+\infty} \delta^{ \pm} L^{* I, I I}(\xi) e^{-\delta \pm \xi \lambda y} \cos (\xi x) d \xi .
\end{gathered}
$$

Taking advantage of Equations (29), (30), (8), and (9), 
we have

$$
\left[\begin{array}{l}
g^{* I, I I}(\xi) \\
L^{* I, I I}(\xi)
\end{array}\right]=\left[\begin{array}{l}
M_{1} \\
M_{2}
\end{array}\right] \frac{g^{I, I I}(\xi)}{\xi},
$$

where

$$
\left[\begin{array}{l}
M_{1} \\
M_{2}
\end{array}\right]=\left[\begin{array}{cc}
c_{11}-c_{66} \lambda^{2} & -\left(c_{12}+c_{66}\right) \lambda \\
\left(c_{12}+c_{66}\right) \lambda & c_{66}-c_{22} \lambda^{2}
\end{array}\right]^{-1}\left[\begin{array}{c}
\beta_{1} \\
\beta_{2} \lambda
\end{array}\right]
$$

By the aid of Equations (3)-(5), (25), (26), (29), and (30), the components of stress are in the form of the following expressions:

$$
\begin{aligned}
\sigma_{x}^{I, I I}(x, 0)= & \sum_{j=1}^{2} \int_{0}^{+\infty}\left(c_{11}-c_{12} \gamma_{j} \eta_{j}\right) \xi g_{j}^{I, I I}(\xi) \cos (\xi x) d \xi \\
& +\left(c_{11} M_{1}-c_{12} \lambda M_{2}-\beta_{1}\right) \int_{0}^{+\infty} g^{I, I I}(\xi) \cos (\xi x) d \xi
\end{aligned}
$$

$$
\begin{aligned}
\sigma_{y}^{I, I I}(x, 0)= & \sum_{j=1}^{2} \int_{0}^{+\infty}\left(c_{12}-c_{22} \gamma_{j} \eta_{j}\right) \xi g_{j}^{I, I I}(\xi) \cos (\xi x) d \xi \\
& +\left(c_{12} M_{1}-c_{22} \lambda M_{2}-\beta_{2}\right) \int_{0}^{+\infty} g^{I, I I}(\xi) \cos (\xi x) d \xi
\end{aligned}
$$

$$
\begin{aligned}
\tau_{x y}^{I, I I}(x, 0)= & -c_{66}\left[\sum_{j=1}^{2} \int_{0}^{+\infty} \delta^{ \pm}\left(\gamma_{j}+\eta_{j}\right) \xi g_{j}^{I, I I}(\xi) \sin (\xi x) d \xi\right. \\
& \left.+\int_{0}^{+\infty} \delta^{ \pm}\left(M_{1} \lambda+M_{2}\right) g^{I, I I}(\xi) \sin (\xi x) d \xi\right] .
\end{aligned}
$$

In order to get the explicit solution of this considered problem, we depict the dual integral equations as

$$
\begin{gathered}
\tau_{x y}^{I}(x, 0)=\tau_{x y}^{I I}(x, 0)=0, \quad x>0, \\
v^{I}(x, 0)=-v^{I I}(x, 0)=0, \quad 0<x<a \text { or } x>b .
\end{gathered}
$$

Using Equations (36) and (37), one gets

$$
\begin{gathered}
g_{j}^{I}(\xi)=g_{j}^{I I}(\xi), \\
g_{2}^{I}(\xi)=-\frac{\gamma_{1}+\eta_{1}}{\gamma_{2}+\eta_{2}} g_{1}^{I}(\xi) .
\end{gathered}
$$

Applying Equations (20) and (37), one obtains

$$
\begin{gathered}
\int_{0}^{+\infty} \xi g_{1}^{I}(\xi) \cos (\xi x) d \xi=0, \quad 0<x<a \text { or } x>b, \\
\int_{0}^{+\infty} \xi g_{1}^{I}(\xi) \cos (\xi x) d \xi=\frac{\sigma_{1} x}{\ell_{1}(b-a)}, \quad a<x<b,
\end{gathered}
$$

where

$$
\ell_{1}=\left(c_{12}-c_{22} \gamma_{1} \eta_{1}\right)-\frac{\gamma_{1}+\eta_{1}}{\gamma_{2}+\eta_{2}}\left(c_{12}-c_{22} \gamma_{2} \eta_{2}\right)
$$

In order to solve Equations (39) and (40), the auxiliary function $\phi(x)$ is introduced as

$$
\phi(x)=\frac{\partial v^{I}(x, 0)}{\partial x}
$$

Applying inverse Fourier transform leads to

$$
g_{1}^{I}(\xi) \xi=-\frac{2\left(\gamma_{2}+\eta_{2}\right)}{\left(\eta_{1} \gamma_{2}-\eta_{2} \gamma_{1}\right) \pi} \int_{a}^{b} \phi(s) \sin (\xi s) d s
$$

Inserting Equation (43) into (40), one has

$$
\frac{2}{\pi} \int_{a}^{b} \phi(s) d s \int_{0}^{\infty} \sin (\xi s) \cos (\xi x) d \xi=-\frac{\sigma_{1}\left(\eta_{1} \gamma_{2}-\eta_{2} \gamma_{1}\right)}{\ell_{1}\left(\gamma_{2}+\eta_{2}\right)(b-a)} x .
$$

Recalling the known result [27],

$$
\int_{0}^{\infty} \cos (\xi x) \sin (\xi s) d \xi=\frac{s}{s^{2}-x^{2}} .
$$

Based on Equation (45), Equation (44) can be expressed as

$$
\frac{1}{\pi} \int_{a}^{b} \frac{2 s \phi(s)}{s-x} d s=-\frac{\sigma_{1}\left(\eta_{1} \gamma_{2}-\eta_{2} \gamma_{1}\right)}{\ell_{1}\left(\gamma_{2}+\eta_{2}\right)(b-a)} x
$$

It is convenient to introduce $s^{2}=\bar{s}, x^{2}=\bar{x}, 2 s d s=d \bar{s}, a^{2}$ $=\bar{a}, b^{2}=\bar{b}$, and $\bar{\phi}(\bar{s})=\phi(s)$. Equation (46) is rewritten as

$$
\frac{1}{\pi} \int_{\bar{a}}^{\bar{b}} \frac{\bar{\phi}(\bar{s})}{\bar{s}-\bar{x}} d \bar{s}=\frac{\sigma_{1}\left(\eta_{1} \gamma_{2}-\eta_{2} \gamma_{1}\right)}{\ell_{1}\left(\gamma_{2}+\eta_{2}\right)(b-a)} \sqrt{\bar{x}}
$$

According to the singular integral containing the Cauchy kernel4 [28], the solution of Equation (47) is obtained

$$
\bar{\phi}(\bar{x})=\frac{1}{\pi \sqrt{(\bar{x}-\bar{a})(\bar{b}-\bar{x})}} \int_{\bar{a}}^{\bar{b}} \frac{\sqrt{\bar{s}(\bar{s}-\bar{a})(\bar{b}-\bar{s})}}{\bar{x}-\bar{s}} \frac{\sigma_{1}\left(\eta_{1} \gamma_{2}-\eta_{2} \gamma_{1}\right)}{\ell_{1}\left(\gamma_{2}+\eta_{2}\right)(b-a)} d \bar{s} .
$$

In the application of Equation (42), the closed form of 
elastic displacement is obtained

$$
v^{I}(x, 0)=\int_{\bar{a}}^{\bar{x}} \frac{\bar{\phi}(\bar{t})}{2 \sqrt{t}} d \bar{t} .
$$

Inserting Equation (48) into (34), the stress field is obtained as

$$
\begin{aligned}
\sigma_{y}^{I, I I}(x, 0)= & -\frac{\sigma_{1}}{\pi(b-a) \sqrt{\left(a^{2}-x^{2}\right)\left(b^{2}-x^{2}\right)}} \\
& \times\left[\frac{2 x^{2}\left(x^{2}-a^{2}-b^{2}\right)}{b} F(\lambda)+2 b x^{2} E(\lambda)\right] \\
& +O(1), \quad 0<x<a, \\
\sigma_{y}^{I, I I}(x, 0)= & -\frac{\sigma_{1}}{\pi(b-a) \sqrt{\left(a^{2}-x^{2}\right)\left(b^{2}-x^{2}\right)}} \\
& \times\left[\frac{2 x^{2}\left(x^{2}-a^{2}-b^{2}\right)}{b} F(\lambda)+2 b x^{2} E(\lambda)\right] \\
& +O(1), \quad x>b .
\end{aligned}
$$

$F(\lambda)$ and $E(\lambda)$ denote the first and second kinds of complete elliptical integrals, respectively, where

$$
\lambda=\frac{\sqrt{b^{2}-a^{2}}}{b} .
$$

For simplicity, the detailed procedure of reduction under thermal flux is omitted. The shear stresses are obtained according to [25].

$$
\begin{aligned}
\tau_{x y}^{I, I I}(x, 0)= & \frac{c_{66} H_{2} P}{c_{22}\left(\gamma_{2} \eta_{2}-\gamma_{1} \eta_{1}\right) \sqrt{\left(x^{2}-a^{2}\right)\left(b^{2}-x^{2}\right)}} \\
& \times\left[\frac{4 a^{2} b^{2}}{3}+\frac{\left(a^{2}-b^{2}\right)^{2}}{2}+\frac{2 b^{2}\left(a^{2}+b^{2}\right) E(\lambda)}{3 F(\lambda)}\right. \\
& \left.-\left(2 x^{2}-a^{2}-b^{2}\right)^{2}\right]+O(1), \quad 0<x<a, \\
\tau_{x y}^{I, I I}(x, 0)= & \frac{c_{66} H_{2} P}{c_{22}\left(\gamma_{2} \eta_{2}-\gamma_{1} \eta_{1}\right) \sqrt{\left(x^{2}-a^{2}\right)\left(b^{2}-x^{2}\right)}} \\
& \times\left[\frac{4 a^{2} b^{2}}{3}+\frac{\left(a^{2}-b^{2}\right)^{2}}{2}+\frac{2 b^{2}\left(a^{2}+b^{2}\right) E(\lambda)}{3 F(\lambda)}\right. \\
& \left.-\left(2 x^{2}-a^{2}-b^{2}\right)^{2}\right]+O(1), \quad x>b,
\end{aligned}
$$

where

$$
\begin{gathered}
P=\frac{\left(Q_{1}-Q_{1 c}\right) \ell_{2}}{(b-a)^{2}(b+a) \lambda \lambda_{y}}, \ell_{2}=\frac{H_{1}}{H_{2}}, H_{1} \\
=\left(\gamma_{1}+\eta_{1}\right)\left(c_{22} \gamma_{2} \eta_{2} M_{1}-c_{22} \lambda M_{2}-\beta_{2}\right) \\
+\left(\gamma_{2}+\eta_{2}\right)\left(c_{22} \lambda M_{2}+\beta_{2}-c_{22} \gamma_{1} \eta_{1} M_{1}\right) \\
+c_{22}\left(M_{1} \lambda+M_{2}\right)\left(\gamma_{1} \eta_{1}-\gamma_{2} \eta_{2}\right) \\
H_{2}=\left(\gamma_{1}+\eta_{1}\right)\left(c_{22} \gamma_{2} \eta_{2}-c_{12}\right) \\
+\left(\gamma_{2}+\eta_{2}\right)\left(c_{12}-c_{22} \gamma_{1} \eta_{1}\right) .
\end{gathered}
$$

By superposition theory, the exact solutions of the physical quantities are obtained subject to linear thermal flux $\left(-Q_{1} x / 2(b-a)^{2}\right)$ and linear mechanical load $\left(\sigma_{1}|x| /(b-a)+\sigma_{0}\right)$.

3.3. Crack-Tip Field. Using Equations (2) and (23), one obtains the closed form of heat flux to the crack surface.

$$
Q_{1 c}=\frac{2 \varepsilon Q_{1}(b-a)^{2} \lambda+Q_{1} R_{c} \sqrt{\left(x^{2}-a^{2}\right)\left(b^{2}-x^{2}\right)}}{2(b-a)^{2} \lambda+R_{c} \sqrt{\left(x^{2}-a^{2}\right)\left(b^{2}-x^{2}\right)}}
$$

We define the value of $R_{c}=\lambda_{y} / h_{c}$ to stand for the dimensionless thermal resistance between crack faces. It is easily found Equation (54) is different from that in [25]. When $R_{c}=0$ and $R_{c} \longrightarrow \infty$, one obtains $Q_{1 c}=\varepsilon Q_{1}$ or $Q_{1 c} \longrightarrow Q_{1}$, meaning partially thermally insulated or fully conductive cracks. When $R_{c}=0$ and $\varepsilon=0$, one has $Q_{1 c}=0$, meaning fully thermally insulated cracks.

\section{Fracture Parameters}

It is important that the stress intensity factors including the mode-I and mode-II should be defined as the analysis of cracked growth.

$$
\begin{aligned}
K_{I}^{\text {Inn }} & =\lim _{x \longrightarrow a^{-}} \sqrt{2 \pi(a-x)} \sigma_{y}^{I, I I}(x, 0), K_{I}{ }^{\text {Out }} \\
& =\lim _{x \longrightarrow b^{+}} \sqrt{2 \pi(x-b)} \sigma_{y}^{I, I I}(x, 0), \\
K_{I I}^{\text {Inn }} & =\lim _{x \longrightarrow a^{-}} \sqrt{2 \pi(a-x)} \tau_{x y}^{I, I I}(x, 0), K_{I I}^{\text {Out }} \\
& =\lim _{x \longrightarrow b^{+}} \sqrt{2 \pi(x-b)} \tau_{x y}^{I, I I}(x, 0) .
\end{aligned}
$$

Based on Equation (55), one can obtain

$$
\begin{gathered}
K_{I}^{\text {Inn }}=\sqrt{\frac{\pi}{a\left(b^{2}-a^{2}\right)}}\left\{2 a^{2} b[F(\lambda)-E(\lambda)]\right\} \frac{\sigma_{1}}{\pi(b-a)}, \\
K_{I}^{\text {Out }}=\sqrt{\frac{\pi}{b\left(b^{2}-a^{2}\right)}}\left\{2 b\left[b^{2} E(\lambda)-a^{2} F(\lambda)\right]\right\} \frac{\sigma_{1}}{\pi(b-a)} .
\end{gathered}
$$

When $a=0$, it means the mode-I stress intensity factor 
TABLE 1: Tyrannohex.

\begin{tabular}{cccccccc}
\hline $\begin{array}{c}E_{x x} \\
(\mathrm{MPa})\end{array}$ & $\begin{array}{c}E_{y y} \\
(\mathrm{MPa})\end{array}$ & $\begin{array}{c}G_{x y} \\
(\mathrm{MPa})\end{array}$ & $v_{x y}$ & $v_{y x}$ & $\begin{array}{c}\alpha_{x x} \\
\left(10^{-7} /{ }^{\circ} \mathrm{C}\right)\end{array}$ & $\begin{array}{c}\alpha_{y y} \\
\left(10^{-7} /{ }^{\circ} \mathrm{C}\right)\end{array}$ & $\begin{array}{c}\lambda_{x} \\
\left(\mathrm{w} / \mathrm{m}^{\circ} \mathrm{C}\right)\end{array}$ \\
\hline 135000 & 87000 & 50000 & 0.15 & 0.09667 & 32 & 32 & 3.08 \\
\hline
\end{tabular}

of a single crack with $2 b$. It is easily found that

$$
K_{I}^{\text {Out }}=\frac{2 \sqrt{\pi b} \sigma_{1}}{\pi}
$$

According to Equation (56) and Reference [25], one has

$$
\begin{aligned}
K_{I I}^{\text {Inn }}= & \frac{c_{66} H_{2} P}{2 c_{22}\left(\gamma_{2} \eta_{2}-\gamma_{1} \eta_{1}\right)} \\
& \cdot\left(\frac{4 a^{2} b^{2}}{3}-\frac{\left(a^{2}-b^{2}\right)^{2}}{2}-\frac{2 b^{2}\left(a^{2}+b^{2}\right) E(\lambda)}{3 F(\lambda)}\right) \sqrt{\frac{\pi}{a\left(b^{2}-a^{2}\right)}} \\
K_{I I}^{\text {Out }}= & -\frac{c_{66} H_{2} P}{2 c_{22}\left(\gamma_{2} \eta_{2}-\gamma_{1} \eta_{1}\right)} \\
& \cdot\left(\frac{4 a^{2} b^{2}}{3}-\frac{\left(a^{2}-b^{2}\right)^{2}}{2}-\frac{2 b^{2}\left(a^{2}+b^{2}\right) E(\lambda)}{3 F(\lambda)}\right) \sqrt{\frac{\pi}{b\left(b^{2}-a^{2}\right)}} .
\end{aligned}
$$

The importance of strain energy in a unit volume of the solid is illustrated for nonisothermal $[29,30]$.

$$
\frac{d W}{d V}=\frac{S}{r}
$$

where the definitions of $S$ and $r$ have been given in [24]. For the orthotropic solid, Equation (60) can also be given based on the above concepts of energy density function

$$
\frac{S}{r}=\frac{c_{22}\left(\sigma_{x}^{I}\right)^{2}+c_{11}\left(\sigma_{y}^{I}\right)^{2}-2 c_{12} \sigma_{x}^{I} \sigma_{y}^{I}}{2 c_{11} c_{22}-c_{12}^{2}}+\frac{\left(\tau_{x y}^{I}\right)^{2}}{2 c_{66}} \text {. }
$$

The following strain energy density factor on the crack line is defined to study crack growth in fracture mechanics [24].

$$
S^{\text {Inn,Out }}=\frac{1}{4 \pi}\left[\frac{c_{22} \ell^{2}+c_{11}-2 c_{12} \ell}{c_{11} c_{22}-c_{12}^{2}}\left(K_{I}^{\text {Inn,Out }}\right)^{2}+\frac{1}{c_{66}}\left(K_{I I}^{\text {Inn,Out }}\right)^{2}\right],
$$

where

$$
\ell=\frac{1}{\ell_{1}} \sum_{j=1}^{2}(-1)^{j} \frac{\gamma_{1}+\eta_{1}}{\gamma_{j}+\eta_{j}}\left(c_{11}-c_{12} \gamma_{j} \eta_{j}\right) .
$$

\section{Numerical Results}

For the sake of simplicity, some numerical examples are employed to demonstrate $R_{c}$ and $\varepsilon$ have great effects on

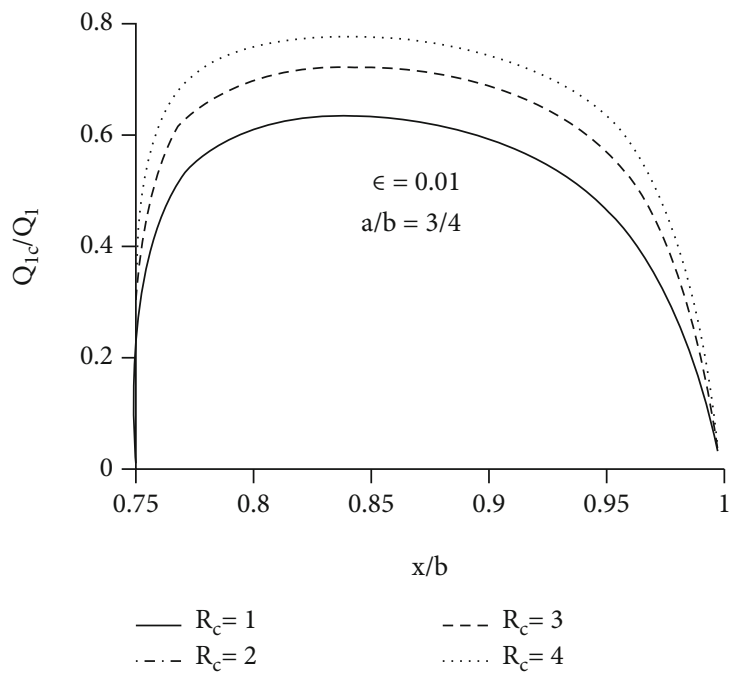

Figure 2: $Q_{1 c} / Q_{1}$ versus $x / b$ with $R_{c}=1,2,3$, 4 for $\varepsilon=0.01$ and $a /$ $b=3 / 4$.

$Q_{1 c}, K_{I I}$, and $S$ subject to linear thermal flux $\left(-Q_{1} x / 2(b-a)^{2}\right.$ ) and linear mechanical load $\left(\sigma_{0}|x| /(b-a)\right)$. The orthotropic material like Tyrannohex is selected as in [31] (Table 1).

Figure 2 shows $Q_{1 c} / Q_{1}$ versus $x / b$ with $R_{c}=1,2,3,4$ for $\varepsilon=0.01$ and $a / b=3 / 4 . Q_{1 c} / Q_{1}$ increases with an increase of $R_{c}$ for a fixed $x / b$ as shown in Figure 2. The case of $R_{c}=1$ implies the heat conduction between the upper and blow crack faces is the same as that of external material of crack. The cases of $R_{c}=2,3,4$ imply to the heat conduction between the upper and blow crack faces are twice, triple, and quadruple as much as thermal conductivities of the external material of crack. Figure 3 displays $Q_{1 c} / Q_{1}$ versus $x / b$ with $\varepsilon=-0.02,0,0.02,0.04$ for $R_{c}=2$ and $a / b=3 / 4$. As the dimensionless thermal resistance $R_{c}$ increases, $Q_{1 c} / Q_{1}$ increases. The constant $\varepsilon$ is considered to be an adjustment quantity. The bigger the value of constant $\varepsilon$, the greater the heat flux per thickness through crack. It means making use of the extended partially insulated crack model involving the greater $R_{c}$ or $\varepsilon$ will overestimate the heat flux per thickness to the crack surface. Furthermore, it is suitable to decease or increase stress field near outer and inn cracks tip by the way of filling certain materials into the region between the upper and below crack faces according to Figures 2 and 3.

Figure 4 displays $K_{I I}^{\text {Inn }} / K_{I I 0}{ }^{\text {Inn }}$ or $K_{I I}$ Out $/ K_{I I 0}$ Out versus $x / b$ with $a / b=0.25,0.5,0.75$ where $K_{I I 0}^{\mathrm{Inn}}$ and $K_{I I 0}^{\text {Out }}$ denote

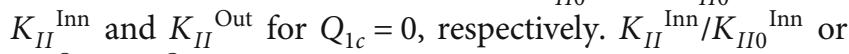
$K_{I I}{ }^{\text {Out }} / K_{I I 0}$ Out decreases when $R_{c}$ increases for a fixed $x / b$. Figure 5 displays $K_{I I}{ }^{\mathrm{Inn}} / K_{I I 0}{ }^{\mathrm{Inn}}$ or $K_{I I}{ }^{\text {Out }} / K_{I I 0}{ }^{\text {Out }}$ versus $x / b$ with $R_{c}=1,2,3,4$ for $\varepsilon=0.01$ and $a / b=3 / 4$. As the 


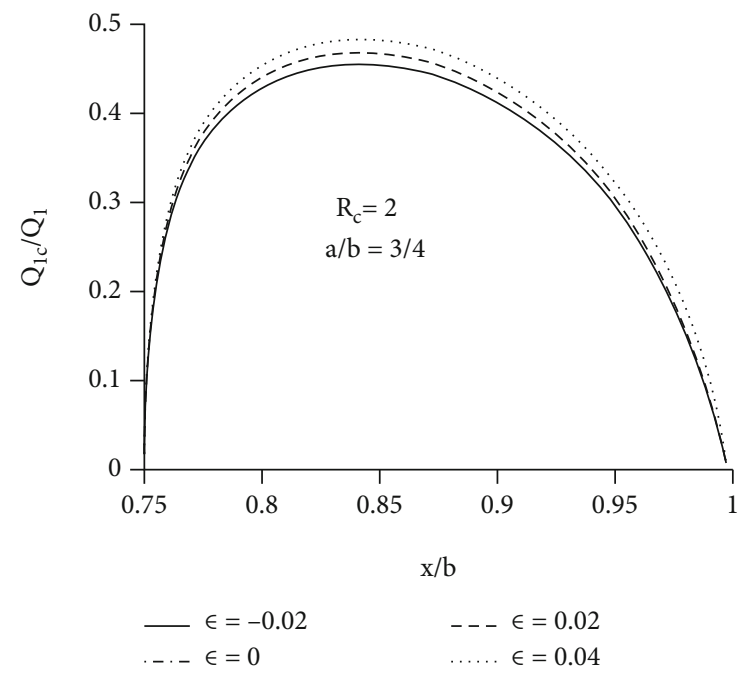

Figure 3: $Q_{1 c} / Q_{1}$ versus $x / b$ with $\varepsilon=-0.02,0,0.02,0.04$ for $R_{c}=2$ and $a / b=3 / 4$.

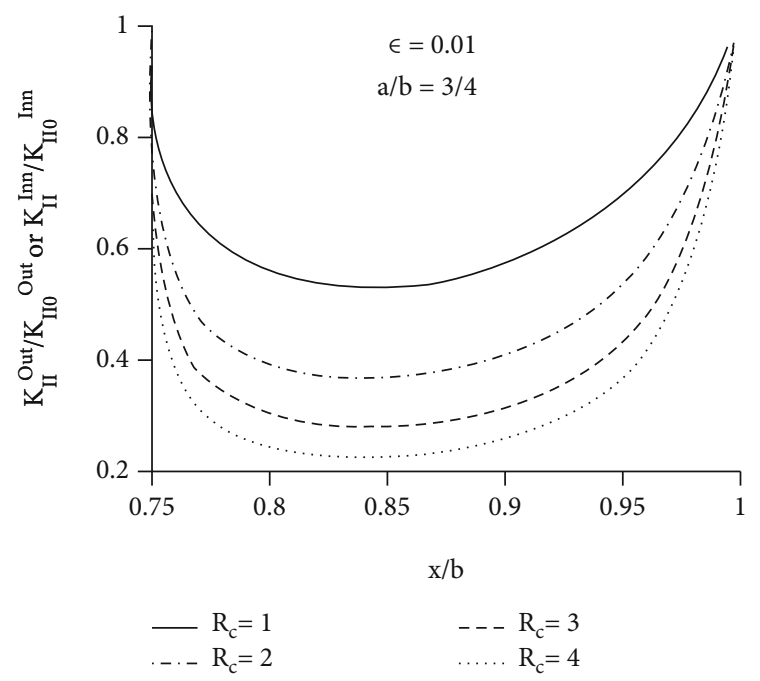

FIgURE 4: $K_{I I}{ }_{I I}^{\text {Inn }} / K_{I I 0}{ }^{\text {Inn }}$ or $K_{I I}{ }^{\text {Out }} / K_{I I 0}{ }^{\text {Out }}$ versus $x / b$ with $R_{c}=1,2$ , 3, 4 for $\varepsilon=0.01$ and $a / b=3 / 4$.

dimensionless thermal resistance $R_{c}$ increases, the mode-II stress intensity factors decrease. The bigger the value of constant $\varepsilon$, the smaller the mode-II stress intensity factors. It means making use of the extended partially insulated crack model involving the greater $R_{c}$ and $\varepsilon$ will underestimate the mode-II stress intensity factors. The obtained results reveal that the crack face boundary conditions, the thermal properties of crack, and the raised coefficients have great influences on the heat flux per thickness to the crack surface and the mode-II stress intensity factors.

In order to present the influence of the thermal properties of crack on $S^{\text {Inn,Out }}$, the value of $S_{0}$ is easily defined.

$$
S_{0}=\frac{c_{22} \ell^{2}+c_{11}-2 c_{12} \ell}{4\left(c_{11} c_{22}-c_{11}^{2}\right)} \sigma_{0}^{2},
$$

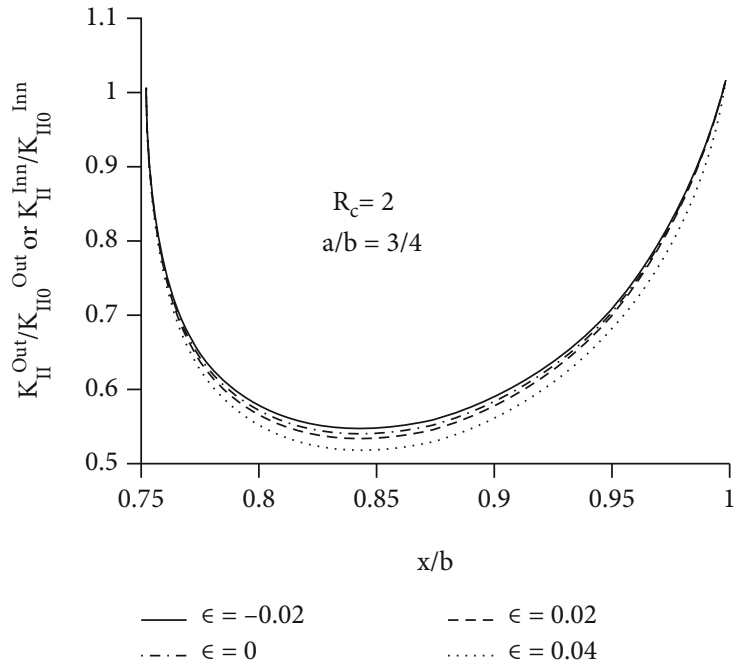

FIgURE 5: $K_{I I}^{\text {Inn }} / K_{I I 0}{ }^{\text {Inn }}$ or $K_{I I}^{\text {Out }} / K_{I I 0}$ Out versus $x / b$ with $\varepsilon=-$ $0.02,0,0.02,0.04$ for $R_{c}=2$ and $a / b=3 / 4$.

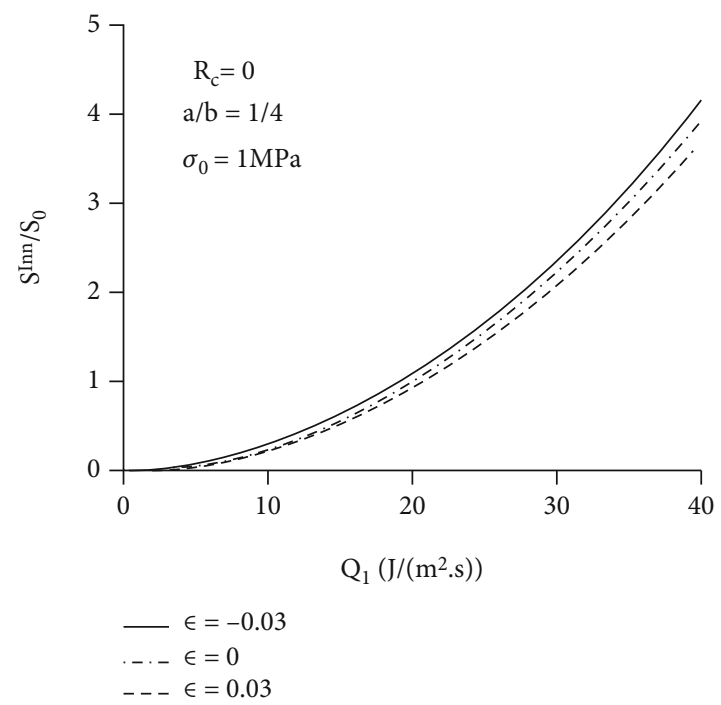

Figure 6: $S^{\mathrm{Inn}} / S_{0}$ versus $Q_{1}$ with $\varepsilon=-0.03,0,0.03$ for $R_{c}=0, \sigma_{0}=1$ $\mathrm{MPa}$, and $a / b=1 / 4$.

which denotes the strain energy density factor of a crack with $2 b$ under mechanical load $\sigma_{0}$. Figures 6 and 7 show $S^{\mathrm{Inn}} / S_{0}$ and $S^{\text {Out }} / S_{0}$ versus $Q_{1}$ with $\varepsilon=-0.03,0,0.03$ for $R_{c}$ $=2, \sigma_{0}=1 \mathrm{MPa}$, and $a / b=1 / 4$. Figures 6 and 7 respond to the two cases of strain energy density factor near outer and inn cracks for partially thermally insulated cracks. It is easily seen that $S^{\mathrm{Inn}} / S_{0}$ and $S^{\text {Out }} / S_{0}$ are made up of the mode-II stress intensity factor under thermal flux $\left(-Q_{1} x / 2(b-a)^{2}\right)$ and the mode-I stress intensity factor induced by mechanical load $\left(\sigma_{0}|x| /(b-a)\right)$. The corresponding $S^{\mathrm{Inn}} / S_{0}$ and $S^{\text {Out }} / S_{0}$ increase with an increase of thermal flux and mechanical load. The strain energy density factor on the crack line is greatly influenced by the adjustment quantity $\varepsilon$. The bigger the value of constant $\varepsilon$, the smaller $S^{\mathrm{Inn}} / S_{0}$ and $S^{\mathrm{Out}} / S_{0}$. So, 


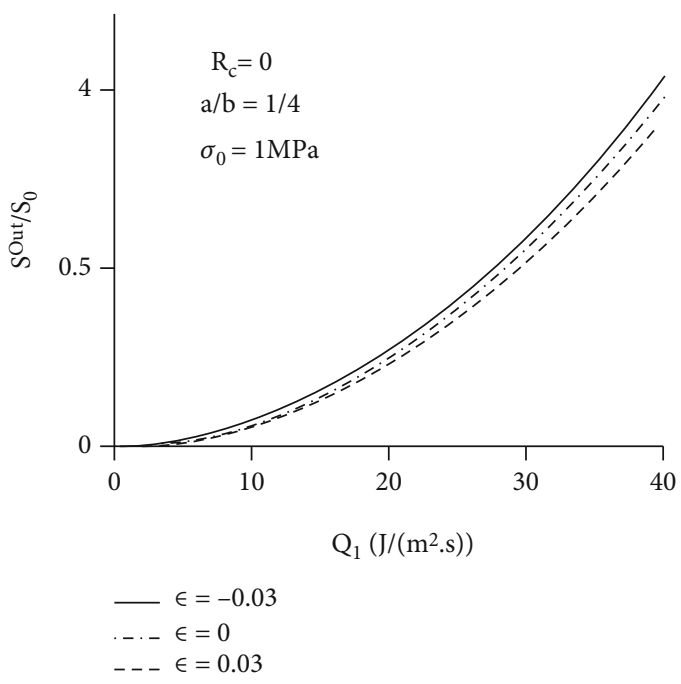

Figure 7: $S^{\text {Out }} / S_{0}$ versus $Q_{1}$ with $\varepsilon=-0.03,0,0.03$ for $R_{c}=0, \sigma_{0}=1$ $\mathrm{MPa}$, and $a / b=1 / 4$.

applying the bigger value of constant $\varepsilon$ will underestimate $S^{\text {Inn }} / S_{0}$ and $S^{\text {Out }} / S_{0}$.

From the above figures, it is revealed $R_{c}$ and $\varepsilon$ have significant impacts on the analysis of a cracked solid. In other words, some physical quantities (i.e., $R_{c}$ and $\varepsilon$ ) should be given enough attention to the analysis of the thermoelastic field.

\section{Conclusions}

This paper addresses two collinear cracks in an orthotropic solid under linear thermal flux and linear mechanical load in this paper. Some physical quantities and fracture parameters are obtained in explicit forms with application of the proposed extended partially insulated crack model, Fourier transform, and superposition theory. The results show that $R_{c}$ and $\varepsilon$ have vital effects on $Q_{1 c}$ and some fracture parameters. The obtained results reveal the boundary conditions of crack face, thermal properties of crack, and the raised coefficients should be concerned about the analysis of a cracked solid under the thermal load.

\section{Conflicts of Interest}

The authors declare that they have no conflicts of interest.

\section{Acknowledgments}

The work was supported by the Hebei University Scientific Research Foundation for higher-level talent (No. 521100221019), the Project Funded by China Postdoctoral Science Foundation (No. 2019M662946), and the Ningxia Key Research and Development Program (Introduction of Talents Project) (No. 2020BEB04039).

\section{References}

[1] W. Nowacki, Thermoelasticity, Pergamon Press, New York, 1962.
[2] R. W. Goldstein and V. M. Vainshelbaum, "Axisymmetric problem of a crack at the interface of layers in a multilayered medium," International Journal of Engineering Science, vol. 14, no. 4, pp. 335-352, 1976.

[3] J. L. Nowinski, Theory of Thermoelasticity with Applications, Sijthoff and Noordhoff, The Netherlands, 1978.

[4] G. C. Sih, "Thermomechanics of solids: nonequilibrium and irreversibility," Theoretical and Applied Fracture Mechanics, vol. 9, no. 3, pp. 175-198, 1988.

[5] Z. Olesiak and I. N. Sneddon, "The distribution of thermal stress in an infinite elastic solid containing a penny-shaped crack," Archive for Rational Mechanics and Analysis, vol. 4, no. 1, pp. 238-254, 1959.

[6] V. I. Fabrikant, Mixed Boundary Value Problem of Potential Theory and Their Applications in Engineering, vol. 42, no. 5, 1991, Kluwer Academic Publishers, The Netherlands, 1991.

[7] Y. M. Tsai, "Orthotropic thermoelastic problem of uniform heat flow disturbed by a central crack," Journal of Composite Materials, vol. 18, no. 2, pp. 122-131, 1984.

[8] H. Ju and R. E. Rowlands, "Mixed-mode thermoelastic fracture analysis of orthotropic composites," International Journal of Fracture, vol. 120, no. 4, pp. 601-621, 2003.

[9] B. X. Chen and X. Z. Zhang, "Thermoelasticity problem of an orthotropic plate with two collinear cracks," International Journal of Fracture, vol. 38, no. 3, pp. 161-192, 1988.

[10] N. Noda, "Transient thermal stress problem in an orthotropic thin plate with a Griffith crack," Archive of Applied Mechanics, vol. 57, no. 3, pp. 175-181, 1987.

[11] A. A. Rizk, "Orthotropic semi-infinite medium with a crack under thermal shock," Theoretical and Applied Fracture Mechanics, vol. 46, no. 3, pp. 217-231, 2006.

[12] J. Kim and G. Paulino, "Mixed-mode fracture of orthotropic functionally graded materials using finite elements and the modified crack closure method," Engineering Fracture Mechanics, vol. 69, no. 14-16, pp. 1557-1586, 2002.

[13] L. M. Brock, "Reflection and diffraction of plane temperaturestep waves in orthotropic thermoelastic solids," Journal of Thermal Stresses, vol. 33, no. 9, pp. 879-904, 2010.

[14] S. Dag, "Thermal fracture analysis of orthotropic functionally graded materials using an equivalent domain integral approach," Engineering Fracture Mechanics, vol. 73, no. 18, pp. 2802-2828, 2006.

[15] B. Wu, D. Peng, and R. Jones, "Fracture analysis for a crack in orthotropic material subjected to combined $2 i$-order symmetrical thermal flux and $2 j$-order symmetrical mechanical loading," Applied Mechanics, vol. 2, no. 1, pp. 127-146, 2021.

[16] X. C. Zhong, X. Y. Long, and L. H. Zhang, "An extended thermal-medium crack model," Applied Mathematical Modelling, vol. 56, pp. 202-216, 2018.

[17] C. H. Wu, "Plane anisotropic thermoelasticity," Journal of Applied Mechanics, vol. 51, no. 4, pp. 724-726, 1984.

[18] M. Nagai, T. Ikeda, and N. Miyazaki, "Stress intensity factor analysis of an interface crack between dissimilar anisotropic materials under thermal stress using the finite element analysis," International Journal of Fracture, vol. 146, no. 4, pp. 233-248, 2007.

[19] L. Liu and G. A. A. Kardomateas, "A dislocation approach for the thermal stress intensity factors of a crack in an infinite anisotropic medium under uniform heat flow," Composites: Part A, vol. 37, no. 7, pp. 989-996, 2006. 
[20] Z. Xue, A. G. Evans, and J. W. Hutchinson, "Delamination susceptibility of coatings under high thermal flux," Journal of Applied Mechanics, vol. 76, no. 4, pp. 728-731, 2009.

[21] Y. T. Zhou, X. Li, and D. H. Yu, "A partially insulated interface crack between a graded orthotropic coating and a homogeneous orthotropic substrate under heat flux supply," International Journal of Solids and Structures, vol. 47, no. 6, pp. 768-778, 2010.

[22] S. Thangitham and H. J. Choi, "Thermal stress singularities in an anisotropic slab containing a crack," Mechanics of Materials, vol. 14, no. 3, pp. 223-238, 1993.

[23] A. Y. Kuo, "Effects of crack surface heat conductance on stress intensity factors," Journal of Applied Mechanics, vol. 57, no. 2, pp. 354-358, 1990.

[24] X. C. Zhong, B. Wu, and K. S. Zhang, "Thermally conducting collinear cracks engulfed by thermomechanical field in a material with orthotropy," Theoretical and Applied Fracture Mechanics, vol. 65, pp. 61-68, 2013.

[25] B. Wu, J. G. Zhu, D. Peng, R. Jones, S. H. Gao, and Y. Y. Lu, "Thermoelastic analysis for two collinear cracks in an orthotropic solid disturbed by antisymmetrical linear heat flow," Mathematical Problems in Engineering, vol. 2017, Article ID 5093404, 10 pages, 2017.

[26] B. Wu, ,D. Peng, and R. Jones, "On the analysis of cracking under a combined quadratic thermal flux and a quadratic mechanical loading," Applied Mathematical Modelling, vol. 68, pp. 182-197, 2019.

[27] I. S. Gradshteyn and I. M. Ryzhik, Table of Integrals, Series, and Products, Elsevier, Academic Press, California, 2007.

[28] N. I. Mushkelishvili, Singular Integral Equations, WoltersNoorhoff, Groningen, 1953.

[29] G. C. Sih, J. Michaopoloulos, and S. C. Chou, Hygrothermoelasticity, Martinus Niijhoof Publishing, Leiden, The Netherland, 1986.

[30] G. C. Sih, Mechanics of Fracture Initiation and Propagation, Kluwer Academic Publishers, Boston, MA, USA, 1991.

[31] S. Itou, "Thermal stress intensity factors of an infinite orthotropic layer with a crack," International Journal of Fracture, vol. 103, no. 3, pp. 279-291, 2000. 